\title{
MicroRNA-203a-3p is a candidate tumor suppressor that targets thrombospondin 2 in colorectal carcinoma
}

\author{
ZHENYUAN QIAN ${ }^{1-3}$, LIJIE GONG $^{4}$, YIPING MOU ${ }^{2,3}$, YONG HAN $^{5}$ and SHUSEN ZHENG ${ }^{1,6}$ \\ ${ }^{1}$ Zhejiang University School of Medicine, Hangzhou, Zhejiang 310058; ${ }^{2}$ Department of Gastrointestinal and \\ Pancreatic Surgery, Zhejiang Provincial People's Hospital, People's Hospital of Hangzhou Medical College; \\ ${ }^{3}$ Key Laboratory of Gastroenterology of Zhejiang Province, Hangzhou, Zhejiang 310014; \\ ${ }^{4}$ Department of Breast Surgery, Zhejiang Cancer Hospital, Hangzhou, Zhejiang 310022; ${ }^{5}$ Clinical Research \\ Institute of Zhejiang Provincial People's Hospital, People's Hospital of Hangzhou Medical College, \\ Hangzhou, Zhejiang 310014; ${ }^{6}$ Division of Hepatobiliary and Pancreatic Surgery, Department of Surgery, \\ The First Affiliated Hospital, School of Medicine, Zhejiang University, Hangzhou, Zhejiang 310003, P.R. China
}

Received February 11, 2019; Accepted August 16, 2019

DOI: $10.3892 /$ or.2019.7310

\begin{abstract}
The aim of the present study was to investigate the role of miR-203a-3p in colorectal cancer (CRC) and identify the target gene of microRNA (miR)-203a-3p. A total of 59 sets of cancer tissues and corresponding adjacent non-tumor tissues were collected from CRC patients (aged 31-78 years) between October 2016 and May 2017. Total RNA extraction and reverse transcription-quantitative polymerase chain reaction analysis, transfection assay, and Transwell and apoptosis assays, western blot analysis, a luciferase reporter assay and immunohistochemistry were performed. miR-203a-3p was found to be significantly downregulated in CRC tissues compared with adjacent normal tissues. The overexpression of miR-203a-3p was shown to inhibit the invasion and migration of human CRC SW480 and HT29 cells, and increase their apoptosis rates. Furthermore, miR-203a-3p downregulated the expression of thrombospondin 2 (THBS2) in SW480 and HT29 cells. It was also experimentally demonstrated that miR-203a-3p binds to the 3'-untranslated region of THBS2, downregulating THBS2 expression and thereby inhibiting CRC progression and metastasis. The expression of miR-203a-3p, which serves a tumor-suppressive role, in CRC tissues was significantly downregulated. As miR-203a-3p was determined to target THBS2 to inhibit CRC progression and metastasis;
\end{abstract}

Correspondence to: Professor Shusen Zheng, Division of Hepatobiliary and Pancreatic Surgery, Department of Surgery, The First Affiliated Hospital, School of Medicine, Zhejiang University, 79 Qingchun Road, Hangzhou, Zhejiang 310003, P.R. China

E-mail: shusenzheng@zju.edu.cn

Key words: microRNA-203a-3p, thrombospondin-2, colorectal cancer, target gene, invasion, migration thus, miR-203a-3p may be considered as a potential novel approach to treating CRC.

\section{Introduction}

Colorectal cancer (CRC) is the third leading cause of cancer-related mortality worldwide, with $\sim 1.7$ million newly diagnosed CRC cases and 800,000 deaths annually $(1,2)$. The most common treatment for CRC is surgery, which has a high success rate when patients are diagnosed early; however, the 5 -year overall survival rate remains unsatisfactory, as the majority of CRC patients are diagnosed at an advanced stage. Consequently, identifying novel biomarkers and therapeutic targets in CRC is crucial.

MicroRNAs (miRNAs), which contain 22-24 nucleotides, are small non-coding RNAs that affect a number of physiological events (3), often by binding to the 3 '-untranslated region (3'UTR) of target genes, thus inhibiting or causing variations in mRNA transcripts (4-6). The occurrence and progression of several tumor types have been found to be associated with abnormal miRNA expression (7-11). Reportedly, aberrant miRNA-203a-3p expression has been detected in numerous cancers (12-18); however, its role and mechanism of action in CRC remain elusive. Therefore, the present study was conducted to investigate miRNA-203a-3p expression in $\mathrm{CRC}$, and elucidate the mechanism underlying the inhibition of apoptosis and promotion of metastasis in CRC.

THBS2 affects interactions between cells and is a potential tumor suppressor (19-21). Accumulating evidence indicates that THBS2 is associated with CRC $(22,23)$. THBS2, secreted by stromal fibroblasts, endothelial cells and immune cells, and belongs to the THBS family of proteins, was identified in 1991 and its sequence was analyzed in $1997(24,25)$. Subsequently, it was found to be associated with various cancer types $(22,26-30)$. The aim of the present study was to investigate the expression of THBS2 in CRC tissues, and determine its link with the overall survival (OS) and disease-free survival (DFS) in patients with CRC. We evaluated the associations among 
miRNA-203a-3p expression, THBS2 expression and CRC progression, in order to determine whether miRNA-203a-3p and THBS2 may be used as biomarkers and therapeutic targets in patients with CRC.

\section{Materials and methods}

Clinical sample collection. We collected 59 sets of cancer tissues and corresponding adjacent non-tumor tissues that were resected from 26 female and 33 male CRC patients (aged 31-78 years) at the Zhejiang Provincial People's Hospital (China) between October 2016 and May 2017. None of the patients received preoperative radiation or chemotherapy. The study protocol was approved by the Ethics Committee of Zhejiang Provincial People's Hospital. Written informed consent was obtained from all patients prior to participation in the study.

Cell culture. The human CRC cell lines HT29, HCT15, SW480 and SW620, and the normal human colon cell line NCM460, were purchased from the Cell Bank of Shanghai Institute of Cell Biology. All the cells were maintained in RPMI-1640 or minimal essential media (MEM; HyClone; GE Healthcare Life Sciences) containing 10\% fetal bovine serum (FBS) (Biowest SAS France), and cultured in a $5 \% \mathrm{CO}_{2}$ incubator at $37^{\circ} \mathrm{C}$. Cells were passaged at $75 \%$ confluence with $0.02 \%$ EDTA/0.25\% trypsin.

Total RNA extraction and reverse transcription-quantitative polymerase chain reaction ( $R T-q P C R)$ analysis. Total cell RNA was extracted from fresh specimens and cells with TRIzol $^{\circledR}$ (Invitrogen; Thermo Fisher Scientific, Inc.) in accordance with the manufacturer's instructions. RT was performed with SYBR Premix Ex Taq according to manufacturer's protocols (Takara Bio, Inc.). RNU6B and GAPDH were used as endogenous controls. miRNA-203a-3p was reverse-transcribed using the following stem-loop RT primer: 5'-GTCGTATCCAGTGCAGGGTCCGAGGTATTCGCA CTGGATACGACGTTGAA-3'. qPCR was performed using FastStart Essential DNA Green Master (Roche Diagnostics) with miRNA-specific primers (forward, 5'-GUGAAAUGU UUAGGACCACUAG3' and reverse, 5'-AGUGGUCCU AAACAUUUCACUU-3'; U6, forward, 5'-ATTGGAACG ATACAGAGA AGATT-3' and reverse, 5'-GGAACGCTT CACGAATTTG-3'). GAPDH, forward, 5'-ATCGTCCAC CGCAAATGCTTCTA-3' and reverse, 5'-AGCCATGCC AATCTCATCTTGTT-3'. THBS2, forward, 5'-CGTGGA CAATGACCTTGTTG-3' and reverse, 5'-GCCATCGTT GTCATCATCAG-3'. The reaction ran on the ABI 7900HT Sequence Detection System (Applied Biosystems; Thermo Fisher Scientific, Inc.) in the presence of SYBR-Green dye (Toyobo Life Science). qPCR was conducted as follows: $95^{\circ} \mathrm{C}$ for $10 \mathrm{~min}, 40$ cycles at $95^{\circ} \mathrm{C}$ for $10 \mathrm{sec}, 60^{\circ} \mathrm{C}$ for $30 \mathrm{sec}$ and $72^{\circ} \mathrm{C}$ for $10 \mathrm{sec}$. Relative expression levels were calculated using the $2^{-\Delta \Delta \mathrm{Cq}}$ method.

Transfection assay. Prior to transfection, cells were plated (3.0x $10^{5}$ cells per well) and cultured at $37^{\circ} \mathrm{C}$ in 6 -well dishes $\left(15 \times 10^{4}\right.$ cells per well) for $20 \mathrm{~h}$. miRNA-203a-3p mimics (Guangzhou RiboBio Co., Ltd.) were transfected into SW480 cells and HT29 cells, which have a relatively low expression of miRNA-203a-3p compared with the normal colonic cells NCM460 and other CRC cell lines. The negative control group (Guangzhou RiboBio Co., Ltd.) was set up in parallel. Transfection of each siRNA $(50 \mathrm{nM})$ was conducted with Lipofectamine ${ }^{\circledR} 3000$ (Thermo Fisher Scientific, Inc.) according to the manufacturer's instructions (miR-203a-3p mimic, forward, 5'-GUGAAAUGUUUAGGA CCACUAG-3' and reverse, 5'-AGUGGUCCUAAACAUUUC ACUU-3'; scramble miRNA, 5'-CAGUACUUUUGUGUA GUACAA-3'). The cells were collected for the following experiments after $48 \mathrm{~h}$ of transfection.

Transwell assay. The CRC cells were cultured for $24 \mathrm{~h}$ after transfection. Migration and invasion assays were performed with a Transwell assay kit (Corning, Inc.) and invasion chambers (Corning, Inc.). Transfected cells (6x10\%/well for migration and $1 \times 10^{5} /$ well for invasion assays) were plated in the upper chamber, which contained FBS-free MEM; the lower chamber contained MEM supplemented with 10\% FBS. After $48 \mathrm{~h}$, cells that had migrated or invaded through the membrane were fixed with methanol and stained with $0.1 \%$ crystal violet in $5 \% \mathrm{CO}_{2}$ at $37^{\circ} \mathrm{C}$ for $15 \mathrm{~min}$. The cells were photographed under a phase-contrast microscope (Olympus Corporation).

Apoptosis assay. At $24 \mathrm{~h}$ after transfection with miRNA203a-3p mimics or negative controls, the cells were washed with PBS and fixed with $70 \%$ ethanol for $>12 \mathrm{~h}$ at $4^{\circ} \mathrm{C}$. Propidium iodide (PI) staining solution $(500 \mu \mathrm{l})$ was then added to the centrifuged cells $(845 \mathrm{x} \mathrm{g}, 3 \mathrm{~min})$ at room temperature, followed by incubation for $30 \mathrm{~min}$ in the dark at room temperature. Cell apoptosis was analyzed by FACSCalibur flow cytometry (BD Biosciences). The percent of apoptotic cells was obtained from FACSCalibur flow cytometry (BD Biosciences) which was used for further calculation. The Annexin V/PI Apoptosis Detection Kit (Beijing Solarbio Science \& Technology, Co., Ltd.) was used to assess apoptosis.

Western blot analysis. At $48 \mathrm{~h}$ after transfection, the cells were harvested, washed and lysed with lysis buffer (Nanjing KeyGen Biotech Co., Ltd.). The proteins were were quantified using BCA kit (Beyotime Institute of Biotechnology) and were separated with $12 \%$ SDS-PAGE $(20 \mu \mathrm{g} / \mathrm{lane})$, and then transferred to microporous membranes (EMD Millipore). The membranes were blocked with Tris-buffered saline with 3\% bovine serum albumin (Solarbio Science \& Technology Co., Ltd.) for $1 \mathrm{~h}$ at room temperature and incubated with primary antibodies against THBS2 (1:500; PA5-80123; Thermo Fisher Scientific,Inc.) and GAPDH (1:1,000; 10494-1-AP; ProteinTech Group, Inc.) for $1 \mathrm{~h}$ at room temperature. The corresponding HRP-conjugated secondary antibody was applied at a 1:2,000 dilution after the primary antibodies. The membranes were evaluated using the Chemi Doc ${ }^{\mathrm{TM}}$ XRS+ imaging system (Bio-Rad Laboratories, Inc., Hercules, CA, USA).

Luciferase reporterassay. The Renilla vector was established by Ruibo Biotechnology Co.,Ltd.HT29 cells were added to 96-well plates and cultured for $24 \mathrm{~h}$. Renilla was used for normalization. The cells were transfected with THBS2-3'UTR-wild-type 

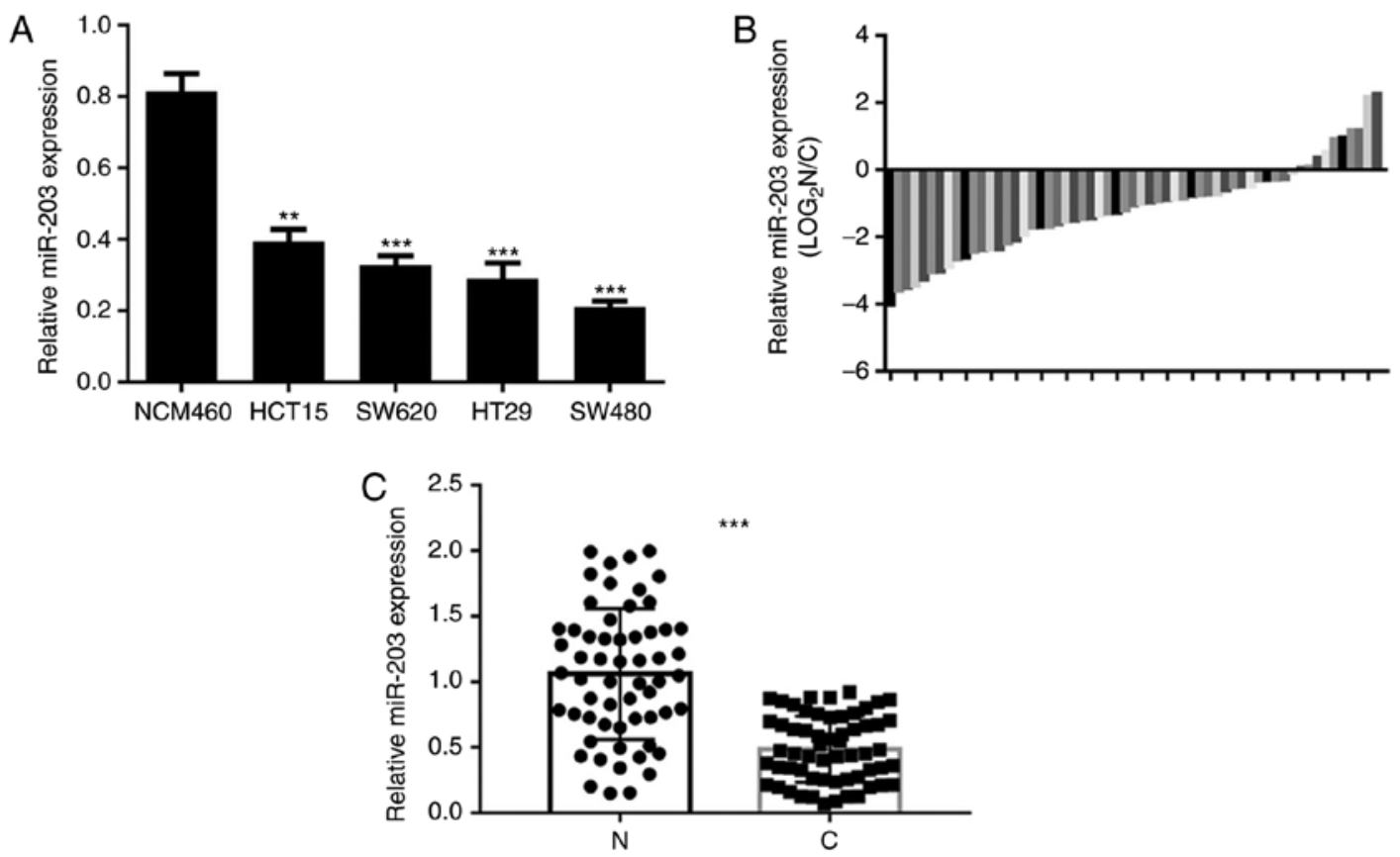

Figure 1. miR-203a-3p was downregulated in CRC tissues and cell lines. (A) miR-203a-3p was downregulated in CRC cell lines. (B) miR-203a-3p was downregulated in 49/59 CRC tissues. (C) microRNA-203a-3p was downregulated in CRC tissues compared with adjacent normal tissues. ${ }^{* *} \mathrm{P}<0.01$ and ${ }^{* * * *} \mathrm{P}<0.001$ vs. NCM460 cells or $\mathrm{N}$ samples. $\mathrm{C}$, cancer; CRC, colorectal cancer; miR, microRNA; N, normal.

(WT) or THBS2-3'UTR-mutant (mut) and miR-203a-3p or miR-control vectors using Lipofectamine 3000. After $48 \mathrm{~h}$, luciferase activity was evaluated by Dual-Luciferase Reporter Assay reagent (Promega Corporation).

Cell proliferation analysis. proliferative ability was detected with A Cell Counting Kit-8 (CCK-8) on the manufacturer's instructions (Dojindo Molecular Technologies, Inc., Kumamoto, Japan). At 24, 48 and $72 \mathrm{~h}$ following transfection, $2 \times 10^{3}$ cells/well were seeded into 96 -well plates and $10 \mu \mathrm{l}$ of CCK- 8 solution was added to assess cell viability. The optical density (OD) was measured using a microplate reader (Molecular Devices LLC) at an absorbance of $450 \mathrm{~nm}$.

Immunohistochemistry. The paraffin-embedded tissue specimens were cut into 5- $\mu \mathrm{m}$ sections. After deparaffinization, antigens were retrieved with $0.01 \mathrm{M}$ citrate buffer ( $\mathrm{pH}$ 6.0) and treated with $3 \% \mathrm{H}_{2} \mathrm{O}_{2}$ for $10 \mathrm{~min}$ at room temperature. The sections were incubated with primary antibody against THBS2 (1:500, PA5-80123; Thermo Fisher Scientific, Inc.) overnight at $4^{\circ} \mathrm{C}$ and then treated with corresponding HRP-conjugated secondary antibody $(1: 2,000)$ for $1 \mathrm{~h}$ at room temperature. After dehydration, the sections were each covered with a single slide. Images were captured with the NanoZoomer Digital Pathology 2.0RS (Hamamatsu Photonics K.K.) and analyzed with NDP.view, version 2.7.25 (Hamamatsu Photonics K.K.). Upright microscope was used in these experiments and the magnification is 200 times.

Bioinformation analysis. We predict the target gene of miRNA with TargetScan (version 5.0; http://genes.mit.edu/targetscan). The level of THBS2 mRNA in the adjacent normal colonic mucosal tissues and CRC tissues and the Kaplan-Meier survival curve analysis of THBS2 in CRC patients in The
Cancer Genome Atlas (TCGA) were analyzed with GEPIA (http://gepia.cancer-pku.cn/).

Statistical analysis. Statistical analysis was conducted using SPSS software version 22.0 (IBM Corp.). Data were presented as the mean \pm standard deviation of experiments repeated in triplicate. Significance between groups was analyzed with a Student's t-test. The correlation between miR-203a-3p and THBS2 expression was examined using Pearson's correlation analysis. Survival analyses were conducted using the Kaplan-Meier method and differences in survival were examined using the log-rank test. $\mathrm{P}<0.05$ was considered to indicate a significant.

\section{Results}

miRNA-203a-3p expression in CRC. The expression of microRNA-203a-3p was found to be significantly lower in the four CRC cell lines (SW480, SW620, HCT15 and HT29) compared with that in the NCM460 human colonic mucosal epithelial cell line (Fig. 1A). Among the CRC cell lines, HCT15 exhibited a relatively high level of miRNA-203a-3p expression. The expression of miRNA-203a-3p in 59 paired CRC and adjacent normal colonic mucosal tissues was detected by RT-qPCR, and was observed to be significantly downregulated in $\mathrm{CRC}$ tissues compared with paired normal tissues (Fig. 1B and C).

miRNA-203-3p affects the invasion and migration potentials of $C R C$ cells. In order to certify the function of miRNA-203a-3p, RT-qPCR was performed to identify the cell lines with lower expression levels of miR-203a-3p. In these cell lines, mimics can effectively activate gene expression. As a result, the HT29 and SW480 cell lines, with lower expression of miRNA-203a-3p 


\section{A}

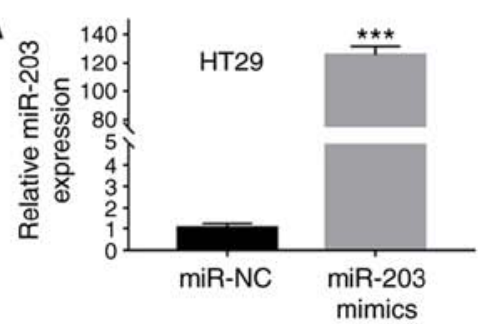

B
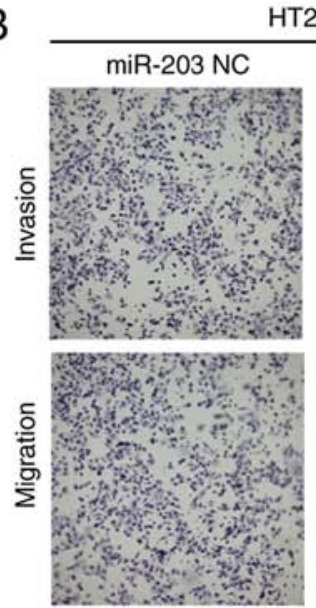

miR-203 mimics
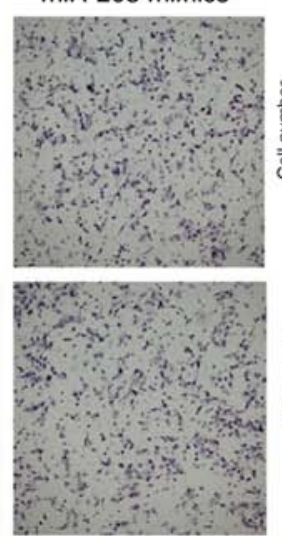
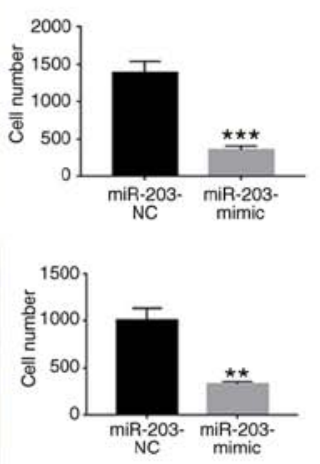
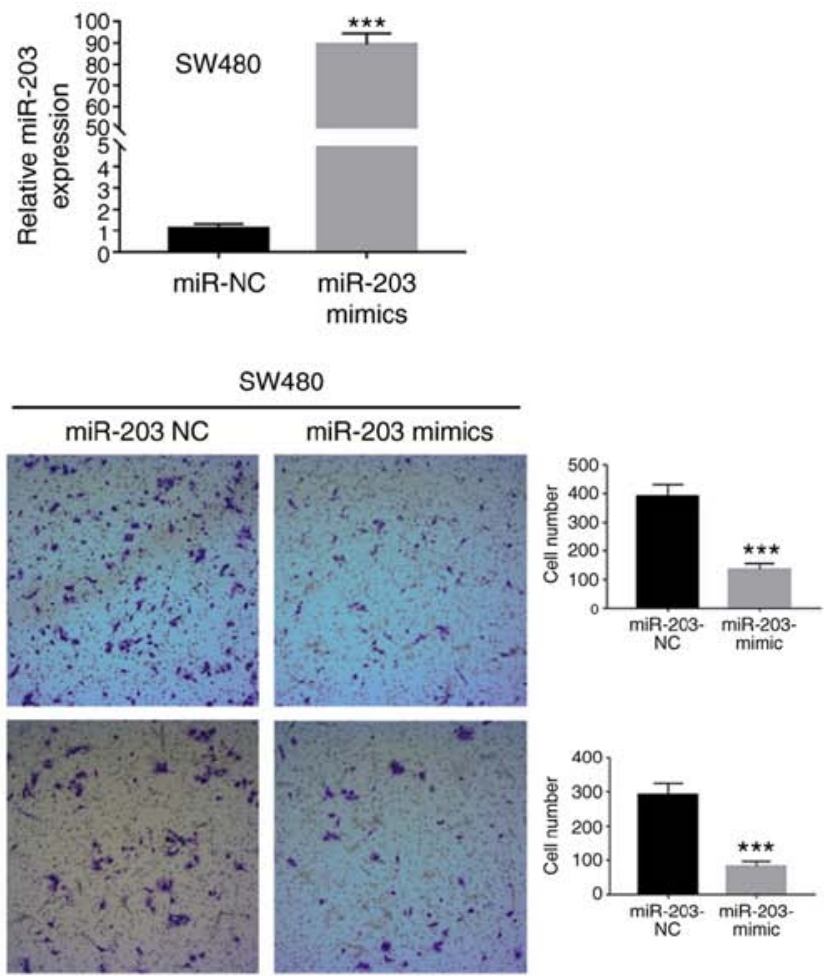

Figure 2. Overexpression of miR-203a-3p can inhibit the invasion and migration of colorectal cancer cells. (A) Reverse transcription-quantitative polymerase chain reaction analysis for evaluation of miR-769 expression in HT29 and SW480 cells transfected with miR-203a-3p mimics or miR-NC. (B) Transwell assay was performed for the analysis of cell invasion and migration. All data were obtained from three independent experiments and presented as mean \pm standard deviation. Magnification, $\mathrm{x} 200 ;{ }^{* *} \mathrm{P}<0.01$ and ${ }^{* * * *} \mathrm{P}<0.001$ vs. miR-NC. miR, microRNA; NC, negative control.

(Fig. 1), were employed for subsequent analysis. The numbers of SW480 and HT29 cells that invaded and migrated across the Transwell membrane were significantly lower for those transfected with miRNA-203a-3p mimic compared with the negative control group (Fig. 2). These data indicate that miRNA-203a-3p affects the invasion and migration potentials of CRC cells.

miRNA-203a-3p affects the apoptosis of CRC cells. miRNA-203a-3p overexpression in HT29 cells was shown to significantly reduce proliferation compared with the control (Fig. 3A). To further investigate the role of miRNA-203a-3p in CRC, HT29 cells were transfected with miRNA-203a-3p mimics. Flow cytometry analysis indicated a significant increase in the apoptotic rate of HT29 cells transfected with miRNA-203a-3p mimics than the control (Fig. 3B).

THBS2 expression in CRC tissues. The protein expression of THBS2 in CRC tissues and paired adjacent normal tissues was detected by immunohistochemical staining, and was found to be notably upregulated in CRC tissues compared with that in the adjacent tissues (Fig. 4A). Furthermore, the mRNA levels of THBS2 in CRC tissues was significantly higher compared with that in the adjacent normal colonic mucosal tissues in TCGA (Fig. 4B). Kaplan-Meier survival curve analysis demonstrated that the OS and DFS of CRC patients with higher THBS2 expression were significantly shorter compared with those of patients with lower THBS2 levels in TCGA (log-rank test, $\mathrm{P}<0.05$; Fig. 4C).
miR-203a-3p suppresses tumor growth and metastasis by epithelial-to-mesenchymal transition and upregulates the expression of Bcl-2-associated X protein (BAX). From western blot analysis, it was determined that HT29 cells transfected with miR-203a-3p exhibited significantly higher expression levels of E-cadherin, and BAX and lower expression of $\mathrm{N}$-cadherin and vimentin compared with the control (Fig. 5).

miR-203a-3p target-gene luciferase reporter assay. The TargetScan Release 5.0 database predicted that THBS2 was a downstream target of miR-203a-3p, and revealed miR-203a-3p target sites within the 3'UTR of THBS2 mRNA (Fig. 6A). A negative correlation was also observed between miR-203a-3p and THBS2 expression in TCGA (Fig. 6B). To verify whether miR-203a-3p binds directly to the 3'UTR region of THBS2, two groups of luciferase reporter constructs were used. Subsequently, cells were co-transfected with miR-203a-3p mimics and two luciferase reporter constructs, one group in the presence of the wild-type (THBS2-wt) 3'UTR, and the other in the presence of the mutant (THBS2-mut) 3'UTR. Luciferase activity in the wt group was significantly lower compared with that in the control group; however, the activity in the mutant luciferase reporter group was markedly unaffected (Fig. 6C). These data indicate that the THBS2 3'UTR contains a specific miR-203a-3p target site. In HT29 cells, overexpression of miR-203a-3p following transfection with miR-203a-3p mimics caused downregulation of THBS2 expression at the protein level (Fig. 6D). These results indicated that THBS2 is a target gene of miR-203a-3p. 

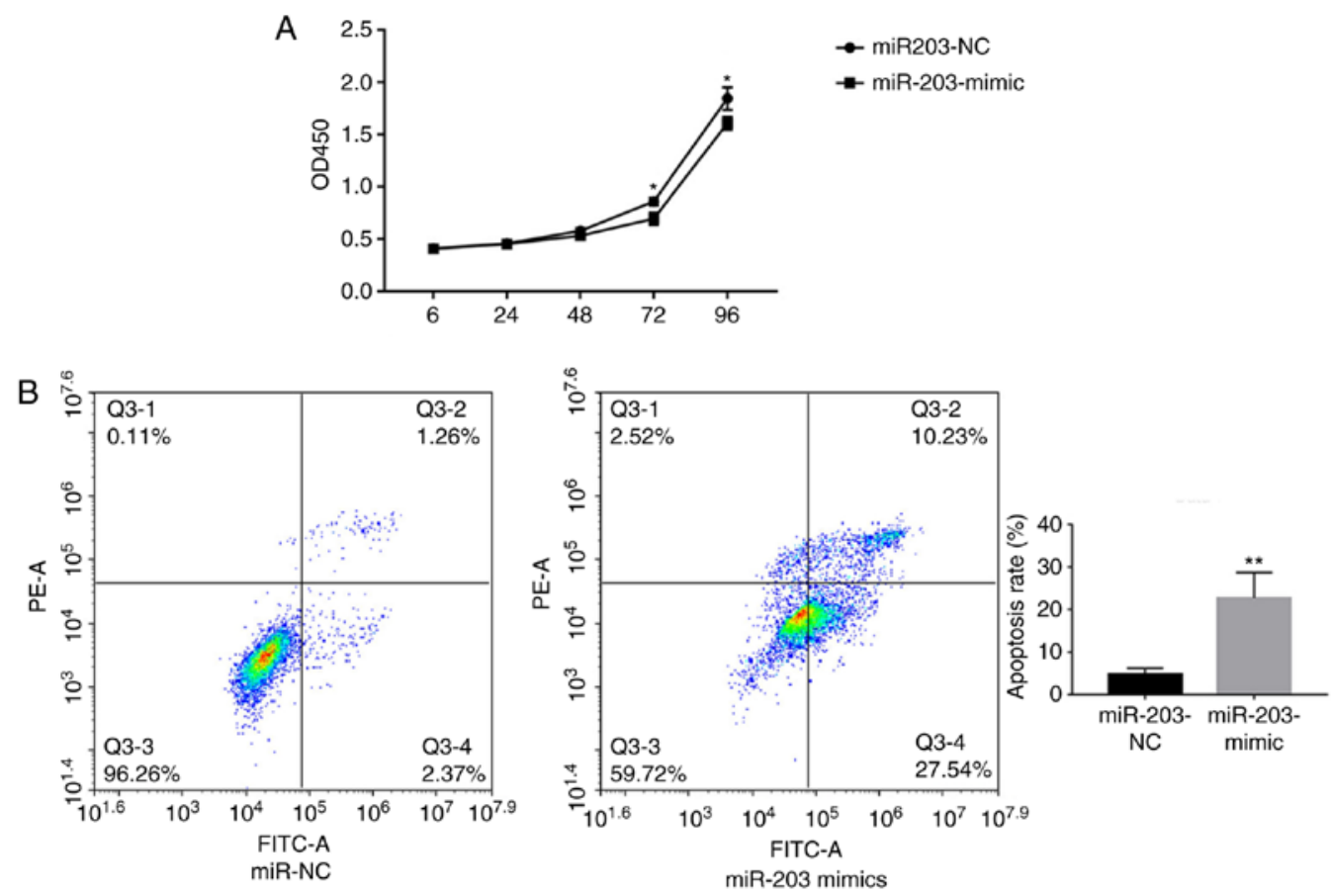

Figure 3. Overexpression of miR-203a-3p can inhibit the proliferation of CRC cells. (A) A Cell Counting Kit-8 assay was used to analyze the proliferation of CRC cells. (B) Cell apoptosis was evaluated by flow cytometry. ${ }^{*} \mathrm{P}<0.05,{ }^{* *} \mathrm{P}<0.01$ vs. miR-203-NC. CRC, colorectal cancer; FITC, fluorescein isothiocyanate; miR, microRNA; NC, negative control; OD, optical density.

A
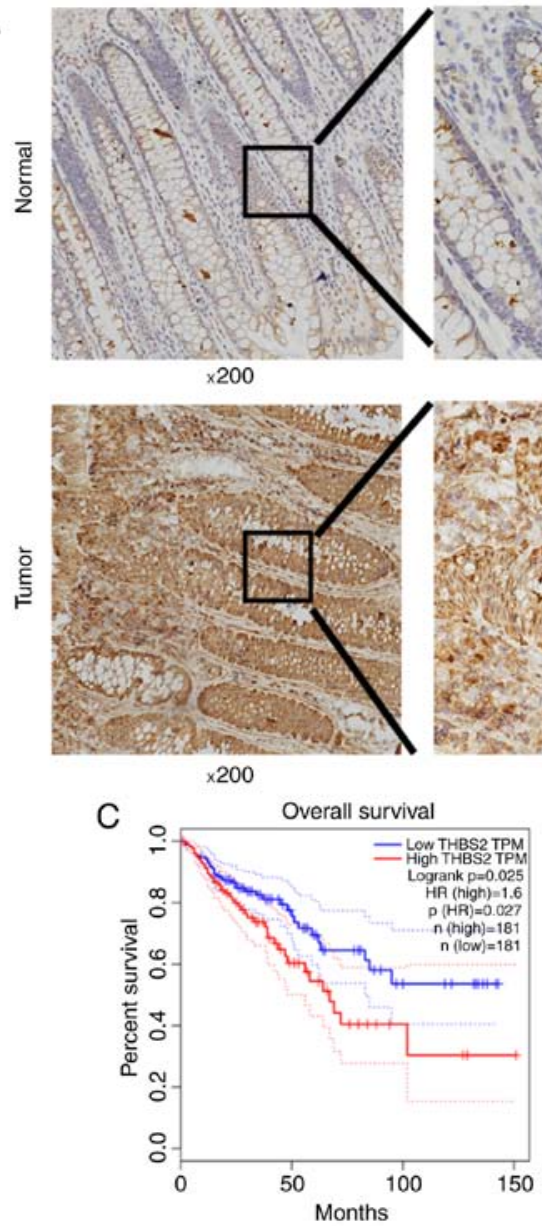

B

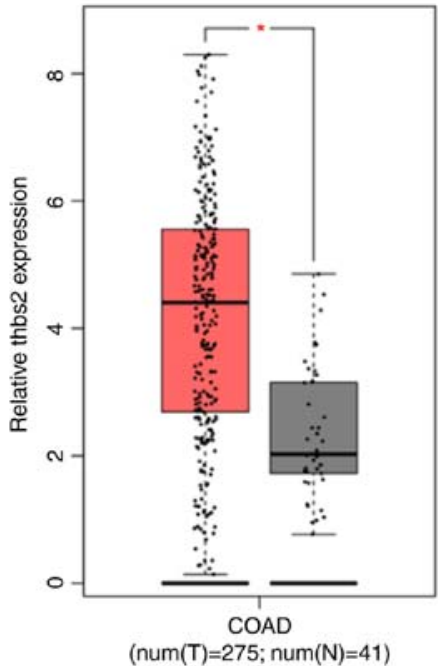

(num $(\mathrm{T})=275 ; \operatorname{num}(\mathrm{N})=41$ )

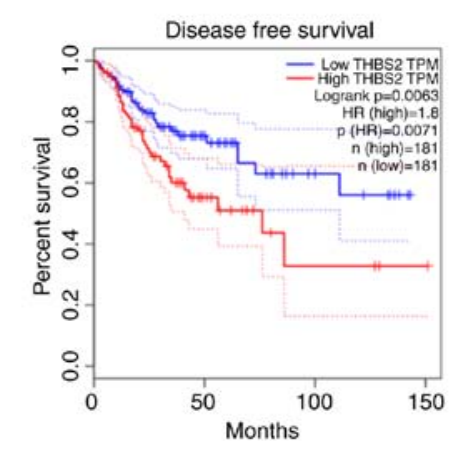

Figure 4. THBS2 is upregulated in CRC tissues and is negatively associated with the prognosis of CRC patients. (A) Immunohistochemistry (magnifications, x200 and x400). (B) Relative THBS2 expression in CRC tissues and adjacent normal tissues in TCGA. (C) Kaplan-Meier survival analysis (overall and disease-free survival rates) based on THBS2 expression levels in CRC tissues in TCGA. "P<0.05. CRC, colorectal cancer; COAD, colonic adenocarcinoma; HR, hazard ratio; TCGA, The Cancer Genome Atlas; THBS2, thrombospondin 2. 

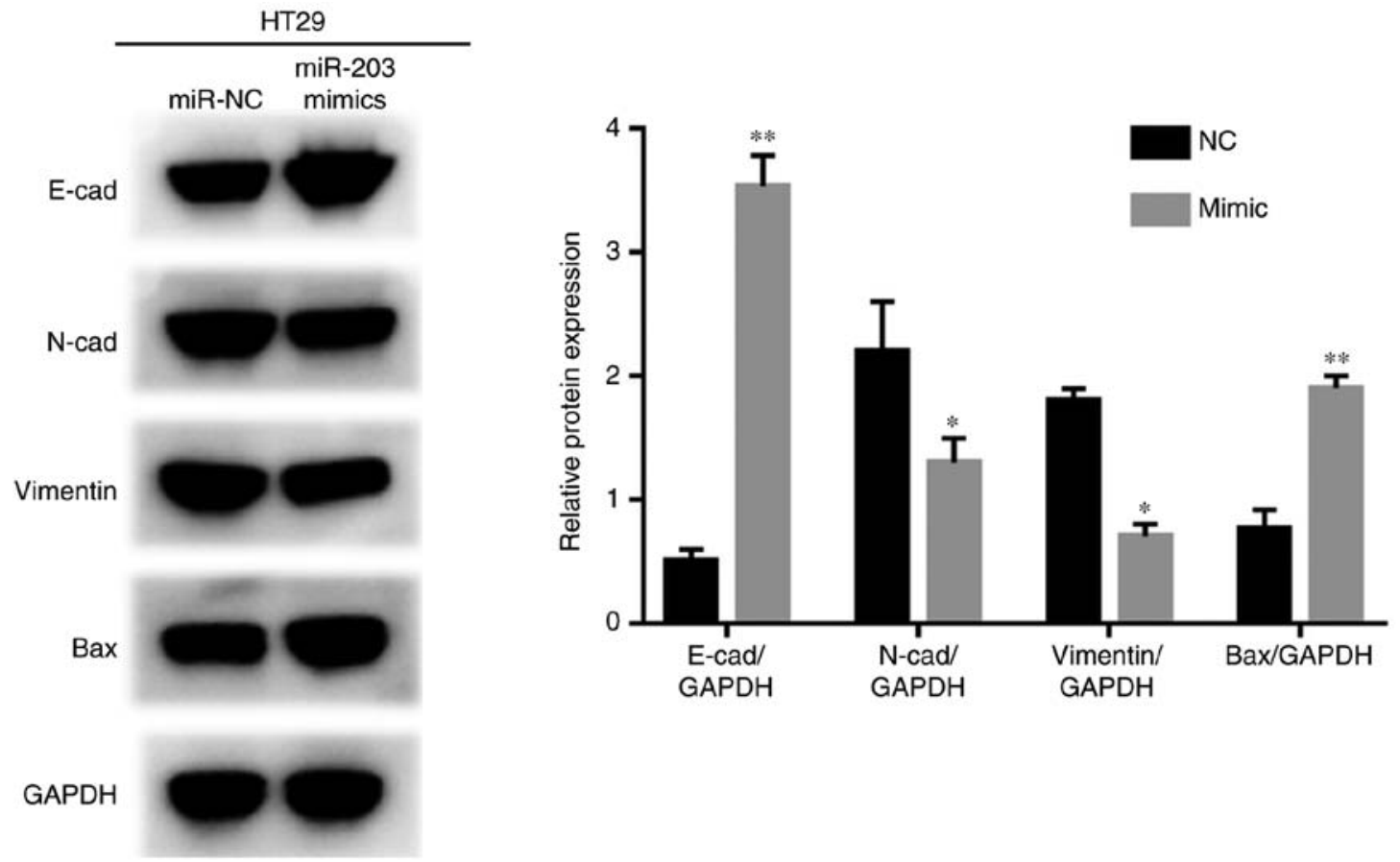

Figure 5. Overexpression of miR-203a-3p can upregulate E-cadherin and Bax, but downregulates vimentin and N-cadherin in HT29 cells. Western blotting was used to determine the protein expression levels in miR-203a-3p-overexpressing HT29 cells. ${ }^{\mathrm{P}}<0.05,{ }^{* *} \mathrm{P}<0.01$ vs. NC. E-cad, E-cadherin; miR, microRNA; $\mathrm{N}$-cad, N-cadherin; NC, negative control.

A

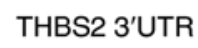

hsa-miR-203

Mutant 3'UTR
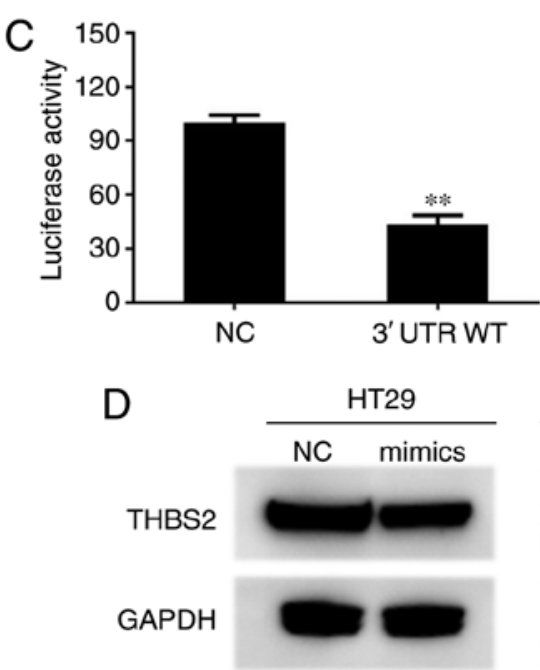

... ACCUgaUUUGaUgauACAUUUCA...

GAUCACCAGGAUUUGUAAAGU

...ACCUGAUUUGAUGAUUGUAAAGU...
B Pearson-correlation:-0.3059

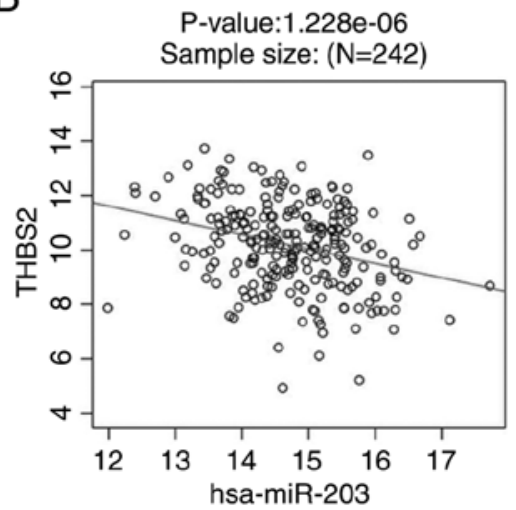

NC

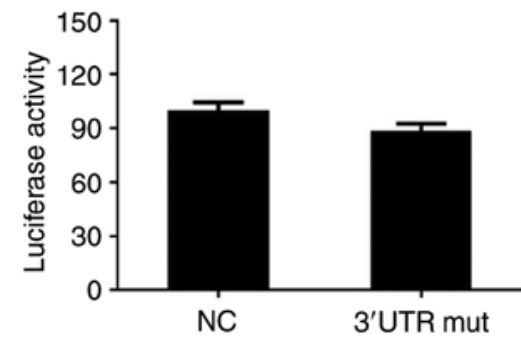

3'UTR mut
SW480

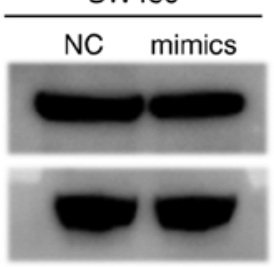

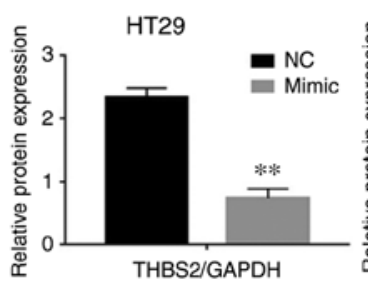

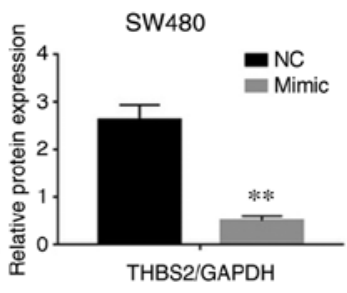

Figure 6. miR-203a-3p binds to the 3'UTR of THBS2. (A) Base pairing of THBS2 and miR-203a-3p, and (B) negative correlation of THBS2 and miR-203a-3p in The Cancer Genome Atlas. (C) Luciferase assay. (D) Western blot analysis of miR-NC and miR-203a-3p-transfected HT29 and SW480 cells. ${ }^{* *} \mathrm{P}<0.01$ vs. NC. 3'UTR, 3'-untranslated region; hsa, homo sapiens; miR, microRNA; NC, negative control; THBS2, thrombospondin 2.

\section{Discussion}

Accumulating evidence has revealed associations between miRNAs and a wide range of tumors $(31,32)$ and, more specifically, between aberrant miRNA-203a-3p expression and tumorigenesis (13-15). miRNA-203a-3p serves different roles in a variety of cancers, depending on the type of the tumor and the targeted genes. 
In the present study, we found that the expression of miRNA-203a-3p in CRC tissues was significantly downregulated compared with that in paired normal tissues, which indicates that miRNA-203a-3p plays a tumor-suppressive role in human CRC. miRNA-203a-3p overexpression was also found to suppress metastatic ability in our study, further confirming that miRNA-203a-3p serves an important role in CRC metastasis. It was also demonstrated that miRNA-203a-3p affected CRC cell apoptosis.

To explore the function of miRNA-203a-3p in CRC and to determine the underlying mechanism, the identification of regulatory targets is crucial. Our western blot results indicated that miRNA-203a-3p promotes tumor metastasis and growth. Overexpression of miRNA-203a-3p expression led to downregulation of $\mathrm{N}$-cadherin, vimentin and the increased expression of E-cad and Bax, which may cause tumor progression. By reviewing miRNA databases, THBS2 was identified as a candidate downstream target of miRNA-203a-3p. THBS2 is secreted by stromal fibroblasts, endothelial cells and immune cells, and it belongs to the THBS family of proteins (24). THBS2 affects interactions between cells and is a potent tumor suppressor with a role in CRC $(22,23)$. THBS2 was found to be significantly upregulated in CRC tissues, and it was demonstrated that its expression was negatively correlated with CRC prognosis.

To verify whether miRNA-203a-3p binds directly to the 3'UTR region of $T H B S 2$, two groups of luciferase reporter constructs were used. Subsequently, cells were co-transfected with miRNA-203a-3p mimics and the two luciferase reporter constructs, one group in the presence of THBS2-wt 3'UTR, and the other in the presence of the THBS2-mut 3'UTR. Luciferase activity in the wt group was found to be significantly lower compared with that in the control group; however, activity in the mutant THBS2 luciferase reporter group was markedly unaffected. These data indicate that the THBS2 3'UTR contains a specific miRNA-203a-3p target site. Combining those results with our findings that miRNA-203a-3p suppresses proliferation, apoptosis and metastasis of CRC cells, it may be concluded that miRNA-203a-3p exerts a suppressive effect on CRC cells through the suppression of THBS2. In the future, we aim to employ an animal model to confirm the results of our in vitro analysis.

In summary, the present study provided evidence that miRNA-203a-3p is downregulated in CRC tissues and cell lines, and affects CRC metastasis by targeting THBS2. These findings suggest that miRNA-203a-3p may act as a tumor suppressor in CRC.

\section{Acknowledgements}

Not applicable.

\section{Funding}

The present study was financed by Grant-in-aid for scientific research from the Zhejiang Provincial Natural Science Foundation for the Youth of China (grant no. LQ18H160023).

\section{Availability of materials and data}

The datasets generated and analyzed in the present study are available from the corresponding author on reasonable request.

\section{Authors' contributions}

SZ made substantial contributions to the study. ZQ, LG, YM and $\mathrm{YH}$ performed the experiments and analyzed the data. ZQ and LG wrote the manuscript. SZ and ZQ helped to revise the manuscript. All the authors have read and approved the final version of this manuscript for publication.

\section{Ethics approval and consent to participate}

All the patients who provided the CRC tissue or other tissue had signed informed consent forms prior to surgery. The use of tissues in the experiment was approved by the Ethics committee of Zhejiang Provincial People's Hospital.

\section{Patient consent for publication}

Not applicable.

\section{Competing interests}

All authors declare that they have no competing interests.

\section{References}

1. Bray F, Ferlay J, Soerjomataram I, Siegel RL, Torre LA and Jemal A: Global cancer statistics 2018: GLOBOCAN estimates of incidence and mortality worldwide for 36 cancers in 185 countries. CA Cancer J Clin 68: 394-424, 2018.

2. Fidler MM, Gupta S, Soerjomataram I, Ferlay J, Steliarova-Foucher E and Bray F: Cancer incidence and mortality among young adults aged 20-39 years worldwide in 2012: A population-based study. Lancet Oncol 18: 1579-1589, 2017.

3. Ameres SL and Zamore PD: Diversifying microRNA sequence and function. Nat Rev Mol Cell Biol 14: 475-488, 2013.

4. Jafri MA, Al-Qahtani MH and Shay JW: Role of miRNAs in human cancer metastasis: Implications for therapeutic intervention. Semin Cancer Biol 44: 117-131, 2017.

5. Friedman RC, Farh KH, Burge CB and Bartel DP: Most mammalian mRNAs are conserved targets of microRNAs. Genome Res 19: 92-105, 2009.

6. Liu X, Chen X, Zeng K, Xu M, He B, Pan Y, Sun H, Pan B, Xu X, $\mathrm{Xu}$ T, et al: DNA-methylation-mediated silencing of miR-486-5p promotes colorectal cancer proliferation and migration through activation of PLAGL2/IGF2/ $\beta$-catenin signal pathways. Cell Death Dis, 2018.

7. Feng L, Jing L, Han J, Wang G, Liu Y, Zhang X, Wang Y, Wang F, Ma $\mathrm{H}$ and Liu Y: MicroRNA 486-3p directly targets BIK and regulates apoptosis and invasion in colorectal cancer cells. Onco Targets Ther 11: 8791-8801, 2018.

8. Han C, Song Y and Lian C: MiR-769 inhibits colorectal cancer cell proliferation and invasion by targeting HEY1. Med Sci Monit 24: 9232-9239, 2018.

9. Mizoguchi A, Takayama A, Arai T, Kawauchi J and Sudo H: MicroRNA-8073: Tumor suppressor and potential therapeutic treatment. PLoS One 13: e0209750, 2018.

10. Yang X, Lou Y, Wang M, Liu C, Liu Y and Huang W: miR675 promotes colorectal cancer cell growth dependent on tumor suppressor DMTF1. Mol Med Rep 19: 1481-1490, 2019.

11. Mazeh H, Mizrahi I, Ilyayev N, Halle D, Brucher B, Bilchik A, Protic M, Daumer M, Stojadinovic A, Itzhak A and Nissan A: The diagnostic and prognostic role of microRNA in colorectal cancer-a comprehensive review. J Cancer 4: 281-295, 2013.

12. DeCastro AJ,Dunphy KA, Hutchinson J, Balboni AL, CherukuriP, Jerry DJ and DiRenzo J: MiR203 mediates subversion of stem cell properties during mammary epithelial differentiation via repression of $\triangle$ NP63alpha and promotes mesenchymal-to-epithelial transition. Cell Death Dis 4: e514, 2013.

13. Hu D, Hu Y, Xu W, Yu H, Yang N, Ni S and Fu R: miR203 inhibits the expression of collagen-related genes and the proliferation of hepatic stellate cells through a SMAD3-dependent mechanism. Mol Med Rep 16: 1248-1254, 2017. 
14. Liang M, Shi B, Liu J, He L, Yi G, Zhou L, Yu G and Zhou X: Downregulation of miR203 induces overexpression of PIK3CA and predicts poor prognosis of gastric cancer patients. Drug Des Devel Ther 9: 3607-3616, 2015.

15. Xu D, Wang Q, An Y and Xu L: MiR203 regulates the proliferation, apoptosis and cell cycle progression of pancreatic cancer cells by targeting Survivin. Mol Med Rep 8: 379-384, 2013.

16. Deng B, Wang B, Fang J, Zhu X, Cao Z, Lin Q, Zhou L and Sun X: MiRNA-203 suppresses cell proliferation, migration and invasion in colorectal cancer via targeting of EIF5A2. Sci Rep 6: 28301, 2016.

17. Xiao Z, Qu Z, Chen Z, Fang Z, Zhou K, Huang Z, Guo X and Zhang Y: LncRNA HOTAIR is a prognostic biomarker for the proliferation and chemoresistance of colorectal cancer via MiR-203a-3p-mediated Wnt/ss-catenin signaling pathway. Cell Physiol Biochem 46: 1275-1285, 2018.

18. Liu S and Feng P: MiR-203 Determines poor outcome and suppresses tumor growth by targeting TBK1 in osteosarcoma. Cell Physiol Biochem 37: 1956-1966, 2015.

19. Burnside MN, Pyatt RE, Hughes A, Baker PB and Pierson CR Complex brain malformations associated with chromosome $6 \mathrm{q} 27$ gain that includes THBS2, which encodes thrombospondin 2 , an astrocyte-derived protein of the extracellular matrix. Pediatr Dev Pathol 18: 59-65, 2015.

20. Zhuo C, Li X, Zhuang H, Tian S, Cui H, Jiang R, Liu C, Tao R and Lin X: Elevated THBS2, COL1A2, and SPP1 expression levels as predictors of gastric cancer prognosis. Cell Physiol Biochem 40: $1316-1324,2016$.

21. Sun R, Wu J, Chen Y, Lu M, Zhang S, Lu D and Li Y: Down regulation of Thrombospondin 2 predicts poor prognosis in patients with gastric cancer. Mol Cancer 13: 225, 2014

22. Fei W, Chen L, Chen J, Shi Q, Zhang L, Liu S, Li L, Zheng L and $\mathrm{Hu}$ X: RBP4 and THBS2 are serum biomarkers for diagnosis of colorectal cancer. Oncotarget 8: 92254-92264, 2017.

23. Wang X, Zhang L, Li W, Sun H, Zhang H and Lai M: THBS2 is a potential prognostic biomarker in colorectal cancer. Sci Rep 6: 33366, 2016.

24. Adolph KW, Liska DJ and Bornstein P: Analysis of the promoter and transcription start sites of the human thrombospondin 2 gene (THBS2). Gene 193: 5-11, 1997.
25. Bornstein P, O'Rourke K, Wikstrom K, Wolf FW, Katz R, Li P and Dixit VM: A second, expressed thrombospondin gene (Thbs2) exists in the mouse genome. J Biol Chem 266: 12821-12824, 1991.

26. Ao R, Guan L, Wang Y and Wang JN: Silencing of COL1A2, COL6A3, and THBS2 inhibits gastric cancer cell proliferation, migration, and invasion while promoting apoptosis through the PI3k-Akt signaling pathway. J Cell Biochem 119: 4420-4434, 2018.

27. Chang IW, Li CF, Lin VC, He HL, Liang PI, Wu WJ, Li CC and Huang CN: Prognostic impact of thrombospodin-2 (THBS2) overexpression on patients with urothelial carcinomas of upper urinary tracts and bladders. J Cancer 7: 1541-1549, 2016.

28. Lin X, Hu D, Chen G, Shi Y, Zhang H, Wang X, Guo X, Lu L, Black D, Zheng XW and Luo X: Associations of THBS2 and THBS4 polymorphisms to gastric cancer in a Southeast Chinese population. Cancer Genet 209: 215-222, 2016.

29. Tsai EA, Gilbert MA, Grochowski CM, Underkoffler LA, Meng H, Zhang X, Wang MM, Shitaye H, Hankenson KD, Piccoli D, et al: THBS2 is a candidate modifier of liver disease severity in alagille syndrome. Cell Mol Gastroenterol Hepatol 2: 663-675, 2016.

30. Wei WF, Zhou CF, Wu XG, He LN, Wu LF, Chen XJ, Yan RM, Zhong M, Yu YH, Liang L and Wang W: MicroRNA-221-3p, a TWIST2 target, promotes cervical cancer metastasis by directly targeting THBS2. Cell Death Dis 8: 3220, 2017.

31. Liu S, Dong H, Dai H, Liu D and Wang Z: MicroRNA-216b regulated proliferation and invasion of non-small cell lung cancer by targeting SOX9, Oncol Lett 15: 10077-10083, 2018.

32. Zhang Y, Su Y, Zhao Y, Lv G and Luo Y: MicroRNA720 inhibits pancreatic cancer cell proliferation and invasion by directly targeting cyclin D1. Mol Med Rep 16: 9256-9262, 2017.

(i) (5) This work is licensed under a Creative Commons Attribution-NonCommercial-NoDerivatives 4.0 International (CC BY-NC-ND 4.0) License. 